\title{
Mild Hydrothermal Synthesis of Ni-Cu Nanoparticles
}

\author{
G. H. Mohamed Saeed, ${ }^{1}$ S. Radiman, ${ }^{1}$ S. S. Gasaymeh, ${ }^{1}$ H. N. Lim, ${ }^{2}$ and N. M. Huang ${ }^{3}$ \\ ${ }^{1}$ School of Applied Physics, Faculty of Science and Technology, National University of Malaysia (UKM), Bangi, 43600 \\ Selangor Darul Ehsan, Malaysia \\ ${ }^{2}$ Faculty of Engineering, Nottingham University, Jalan Broga, Semenyih, 43500 Selangor, Malaysia \\ ${ }^{3}$ Low Dimensional Materials Research Centre, Physics Department, Faculty of Science, University of Malaya, \\ 50603 Kuala Lumpur, Malaysia
}

Correspondence should be addressed to S. Radiman, shahidan@ukm.my

Received 11 October 2009; Accepted 8 May 2010

Academic Editor: Michael Harris

Copyright ( 2010 G. H. Mohamed Saeed et al. This is an open access article distributed under the Creative Commons Attribution License, which permits unrestricted use, distribution, and reproduction in any medium, provided the original work is properly cited.

\begin{abstract}
Magnetic Ni-rich Ni-Cu nanoparticles with $\mathrm{Ni}$ : Cu mass ratio $(S)$ of 2.0 and 2.6 were prepared using a mixture of polyoxyethylene (10) isooctylphenyl ether (Triton X-100) and sodium dodecyl sulfate (SDS) in a mild hydrothermal condition at $95^{\circ} \mathrm{C}$. X-ray diffractometry (XRD) showed that the nanoparticles prepared at $S=2.0$ possessed $\mathrm{Ni}-\mathrm{Cu}$ alloy characteristic whereas the characteristic was absent at $S=2.6$. The XRD data was enhanced by Fourier transform infrared spectroscopy (FTIR) which exhibited metal-metal ( $\mathrm{Ni}-\mathrm{Cu}$ ) band at $455 \mathrm{~cm}^{-1}$. Based on transmission electron microscopy (TEM), the average particle sizes for the nanoparticles prepared at $S=2.0$ and 2.6 were in the range of $19-23 \mathrm{~nm}$. The as-prepared nanoparticles exhibited paramagnetic behaviour measured using a vibrating sample magnetometer (VSM) and the specific saturation magnetization decreased at the higher concentration of $\mathrm{Ni}$.
\end{abstract}

\section{Introduction}

Magnetic materials in the form of nanoparticles have received a lot of attention because of their unique magnetic properties, which are dominated by superparamagnetism. These materials can be used in a broad range of applications, including the production of magnetic fluids. Magnetic fluids are produced by dispersing the superparamagnetic nanoparticles in a carrier liquid. These magnetic fluids have potential application in various modern technologies ranging from medicines and pharmaceuticals to electronics and mechanics [1]. As magnetic properties of nanoparticles are dominated by particle size, it is important to control the particle size, size distribution, as well as the morphology of the particles during the synthesis process.

$\mathrm{Ni}$-based bimetallic nanoparticles containing $\mathrm{Cu}$ have gained considerable interest in the last decade due to the high catalytic conversion and selectivity properties [2] and potential giant magnetoresistance materials [3]. In the recent years, $\mathrm{Ni}-\mathrm{Cu}$ alloy nanoparticles have been proposed as mediator for magnetic fluid hyperthermia [4-6]. Several methods have been reported for the preparation of $\mathrm{Ni}-\mathrm{Cu}$ nanoparticles including sol-gel [7], reduction of mixture of $\mathrm{Ni}$ and $\mathrm{Cu}$ compounds under hydrogen [8], evaporation of $\mathrm{Ni}-\mathrm{Cu}$ alloy and cocondensation with organic solvents [9], water-in-oil microemulsion [10], solvothermal [11], and hydrothermal [12].

In this work, we report on the synthesis of $\mathrm{Ni}-\mathrm{Cu}$ nanoparticles by polymer-surfactant-mediated hydrothermal processing route at a fairly low temperature of $95^{\circ} \mathrm{C}$, in which metal ions were localized by the polymer-surfactant association and reduced the metal ions to nanometer size [12].

\section{Experimental}

All reagents were of analytical grade and used as received from Sigma Aldrich. The synthesis method was similar to that described by Niu et al. [12]. Briefly, $0.67 \mathrm{~g}$ nickel acetate and $0.33 \mathrm{~g}$ copper acetate, which gave a mass ratio $(S)$ of 2.0 , were added into $40 \mathrm{~mL}$ distilled water containing $0.16 \mathrm{~g}$ 


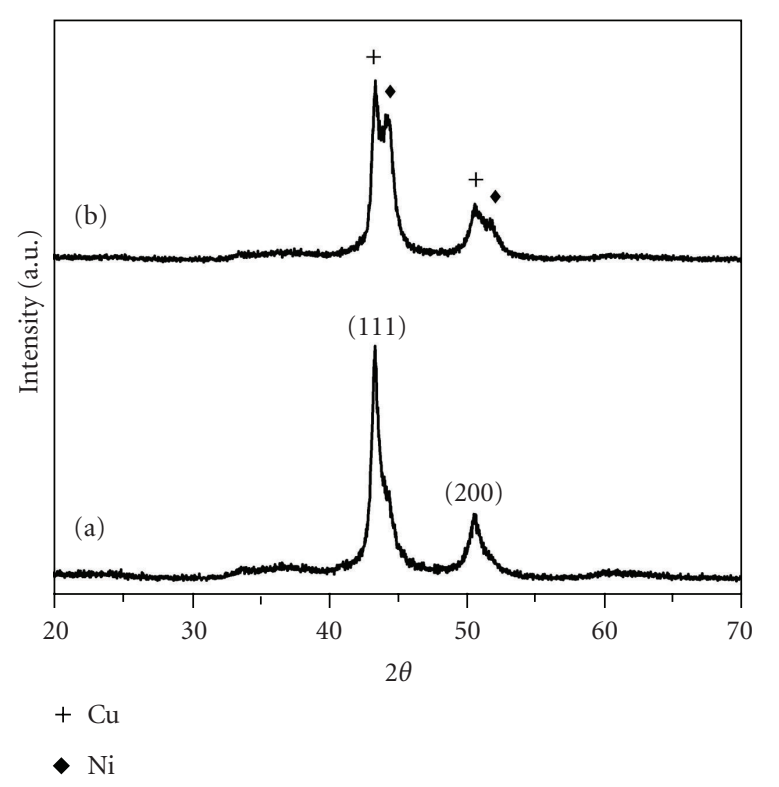

FIgURE 1: XRD patterns of (a) Ni-Cu alloy $(S=2.0)$ and (b) bimetallic $\mathrm{Ni}-\mathrm{Cu}(S=2.6)$.

sodium dodecyl sulfate (SDS) and mixed at room temperature. Then, $1.0 \mathrm{~mL}$ polyoxyethylene (10) isooctylphenyl ether (Triton X-100) and $1.0 \mathrm{~mL}$ ammonia solution were added into the mixture. This was followed by adding $4.0 \mathrm{~mL} 80 \mathrm{wt} \%$ hydrazine hydrate solution into the mixture after strong stirring for 30 minutes. The mild hydrothermal was carried out by pouring the mixture into a SCHOTT glass bottle with $100 \mathrm{~mL}$ capacity, closed tightly and put into an oven at the temperature of $95^{\circ} \mathrm{C}$ for 4 hours. The resulting black precipitate was separated using a centrifuge and washed several times with ethanol and distilled water to remove the remaining surfactants. The product was dried in an oven at $60^{\circ} \mathrm{C}$ for 24 hours. In the same way, the mixture with $S=$ 2.6 was prepared with $0.72 \mathrm{~g}$ of nickel acetate and $0.28 \mathrm{~g}$ of copper acetate.

The samples were characterized by a Bruker AXSD8 Advance X-ray powder diffractometer (XRD) using a scanning rate of $0.025^{\circ} \mathrm{s}^{-1}$ in a $2 \theta$ range from $20^{\circ}$ to $70^{\circ}$ with $\mathrm{Cu} \mathrm{K \alpha}$ radiation $(\lambda=1.54056 \AA)$. The micrographs were taken using a LEO 12CB transmission electron microscopy (TEM). The atomic ratio of $\mathrm{Ni}$ to $\mathrm{Cu}$ was determined by an energy dispersion X-ray analysis (EDXA) which was performed on a LEO 1450 vapour pressure scanning electron microscopy (SEM). The chemical bonding of the samples was observed using a Perkin Elmer GX Model Fourier transforms infrared spectrometer (FTIR). Magnetic hysteresis loops were measured on a vibrating sample magnetometer (VSM) (Lake Shore 4700).

\section{Results and Discussion}

The XRD data of the samples prepared with $S=2.0$ and 2.6 is displayed in Figure 1. Figure 1(a) shows that the sample prepared with $S=2.0$ is an alloy, which is in
TABLe 1: EDXA of Ni-Cu alloy $(S=2.0)$ and bimetallic $\mathrm{Ni}-\mathrm{Cu}(S=$ 2.6).

\begin{tabular}{lcccc}
\hline \multirow{2}{*}{ Element } & \multicolumn{2}{c}{$\mathrm{Ni}-\mathrm{Cu}$ alloy $(S=2.0)$} & \multicolumn{2}{c}{ Bimetallic Ni-Cu $(S=2.6)$} \\
& $\mathrm{Wt} \%$ & Atomic\% & $\mathrm{Wt} \%$ & Atomic\% \\
\hline $\mathrm{Ni}$ & 38.68 & 15.83 & 46.92 & 21.81 \\
$\mathrm{Cu}$ & 20.64 & 7.81 & 20.48 & 8.80 \\
\hline
\end{tabular}

agreement with Niu et al. [12]. The diffraction peaks (111) and (200) are indexed to JCPDS No. 47-1406, which shows the characteristic of an alloy. There were no observable lines in the XRD pattern corresponding to pure $\mathrm{Cu}$ or Ni. On the contrary, the sample prepared with $S=2.6$ shows the XRD pattern of pure $\mathrm{Ni}$ and $\mathrm{Cu}$, which corresponds to JCPDS No. 4-0850 and 4-0836, respectively. Based on the XRD results, an alloy of Ni-Cu could be obtained with $S=2.0$ whereas an alloy does not form at the higher concentration of $\mathrm{Ni}$.

The size of coherently scattering domains (i.e., the crystallite size, $D)$ of $\mathrm{Ni}-\mathrm{Cu}$ alloy $(S=2.00)$ is approximately $8 \mathrm{~nm}$. The calculation is based on the full width at half maximum (FWHM) of diffraction peak from (111) plane using Debye-Scherrer's equation

$$
D=0.98 \lambda \frac{1}{\beta \cos \theta},
$$

where $D_{\mathrm{hkl}}$ is the average crystallite size, $\beta$ is the broadening of FWHM of the main intense peak (111) in radian, $\theta$ is the Bragg angle, and $\lambda$ is the radiation wavelength. The estimated value is much smaller than that of the TEM observation as shown in Figure 2. The particles are uniformly dispersed and spherical in shape. The mean diameter obtained from measurement for $\mathrm{Ni}-\mathrm{Cu}$ alloy $(S=2.0)$ and bimetallic Ni$\mathrm{Cu}(S=2.6)$ is $22.56 \pm 4.32 \mathrm{~nm}$ and $19.97 \pm 3.94 \mathrm{~nm}$, respectively. A plausible explanation is that the nanoparticles are attracted to one another via magnetism, and thus, the attraction aggregates the nanoparticles into much larger particles. The lattice parameter of the $\mathrm{Ni}-\mathrm{Cu}$ alloy $(S=2.0)$ is $3.586 \AA$, which is consistent with Niu et al. [12]. The Ni$\mathrm{Cu}$ ratios are consistent with the values obtained from energy dispersive X-ray analysis (EDXA) as shown in Table 1.

The observed FTIR spectra of the as-synthesized nanoparticles are shown in Figure 3. The bands at 1598, 1375,664 , and $548 \mathrm{~cm}^{-1}$ can be attributed to the vibration characteristic of Triton X-100. The band at $1384 \mathrm{~cm}^{-1}$ is assigned to $-\mathrm{CH}_{3}$ symmetric deformation of Triton X-100. The band at $3420 \mathrm{~cm}^{-1}$ is assigned to the stretching vibration of $-\mathrm{OH}$ group, which may be present due to adsorbed water molecule. The band at $455 \mathrm{~cm}^{-1}$ corresponds to the stretching vibration of metal-metal $(\mathrm{Ni}-\mathrm{Cu})$ band [2]. There is no characteristic band that can be attributed to $\mathrm{Cu}-\mathrm{O}-\mathrm{C}$ or $\mathrm{Ni}-\mathrm{O}-\mathrm{C}$, which suggests that there is no strong interaction between $\mathrm{Ni}-\mathrm{Cu}$ nanoparticles and Triton X-100.

The room temperature magnetic properties of the Ni$\mathrm{Cu}$ nanoparticles, measured in an applied magnetic field of $10^{4} \mathrm{G}$, are depicted in Table 2. From the hysteresis loop shown in Figure 4, squareness $(R)$, the ratio of the remanence to the saturation magnetization is derived to determine 

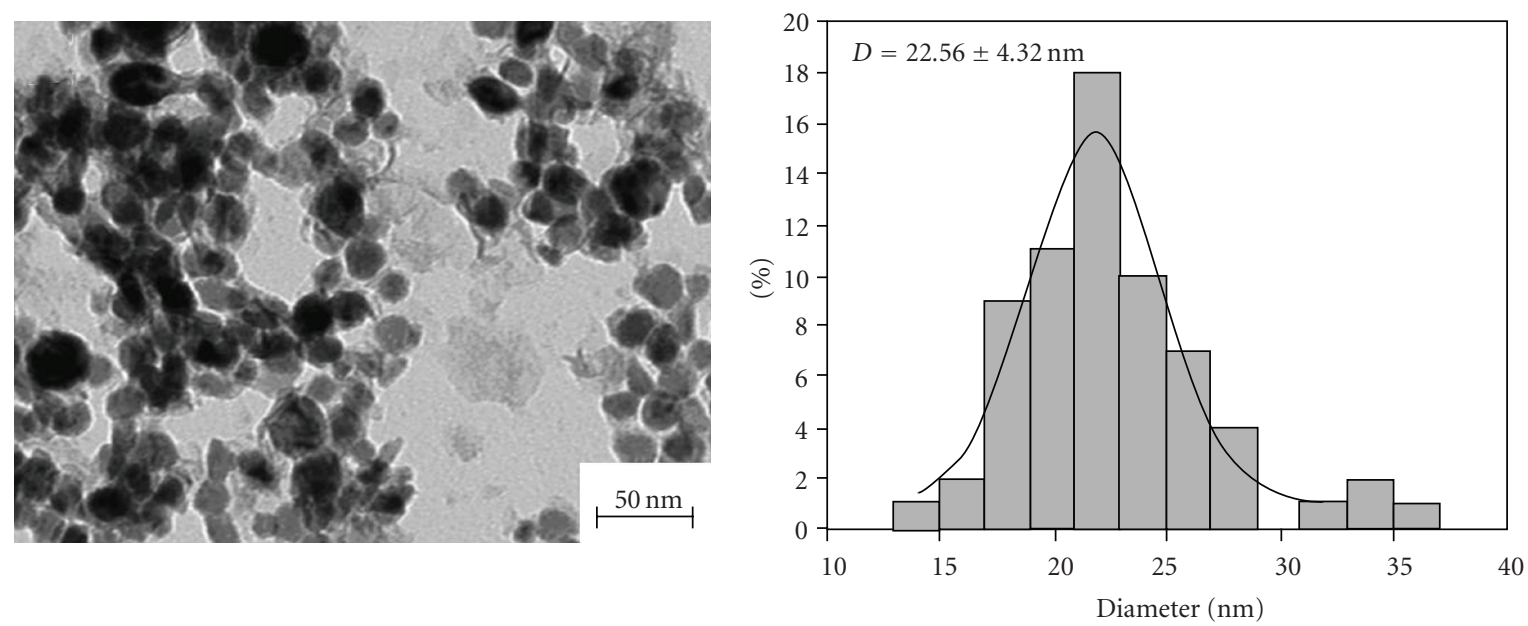

(a)
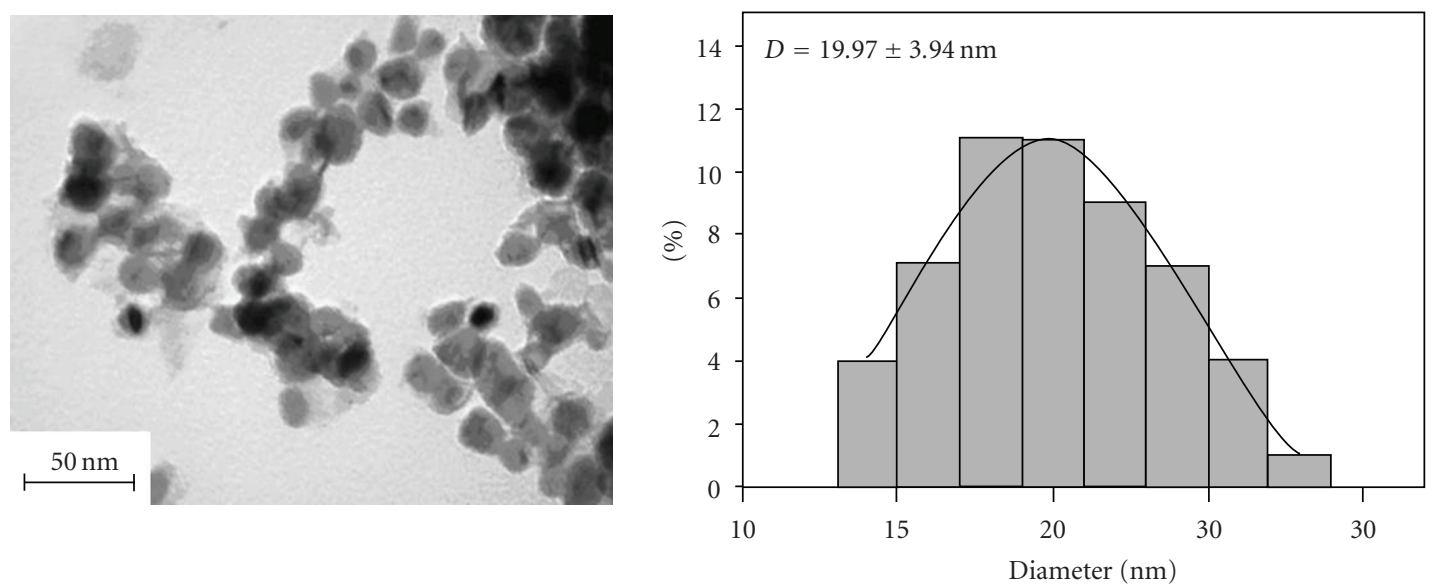

(b)

Figure 2: TEM images (left) and particle size distribution (right) of (a) Ni-Cu alloy $(S=2.0)$ and (b) bimetallic Ni-Cu $(S=2.6$ ).

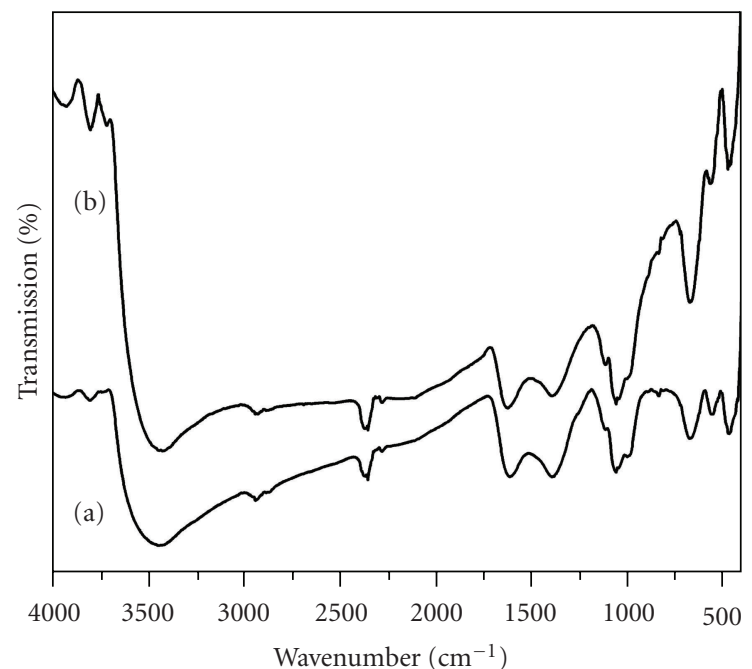

FIGURE 3: FTIR spectra of (a) Ni-Cu alloy $(S=2.0)$ and (b) bimetallic $\mathrm{Ni}-\mathrm{Cu}(S=2.6)$.

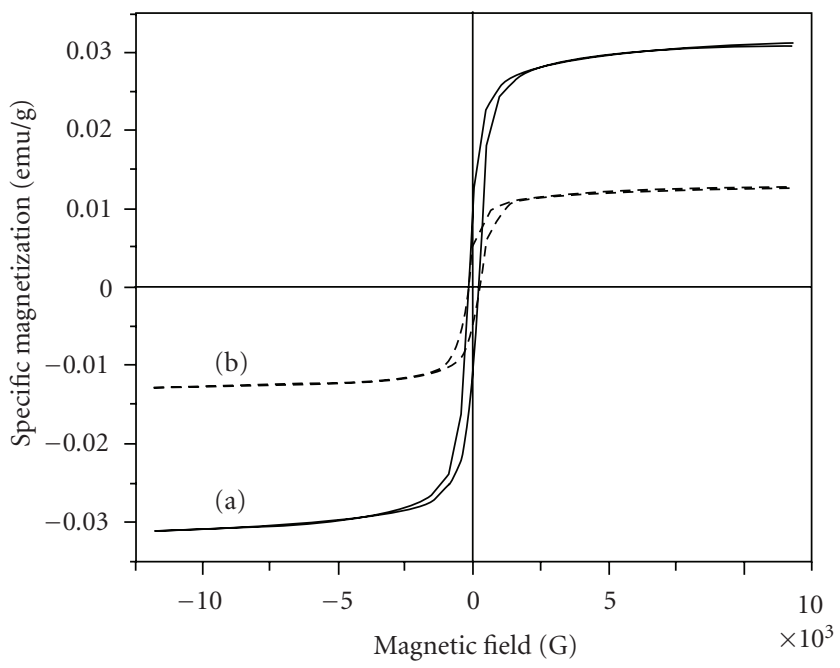

Figure 4: M-H hysteresis loops of (a) Ni-Cu alloy $(S=2.0)$ and (b) bimetallic $\mathrm{Ni}-\mathrm{Cu}(S=2.6)$. 
TAble 2: Magnetic properties of $\mathrm{Ni}-\mathrm{Cu}$ alloy $(S=2.0)$ and bimetallic $\mathrm{Ni}-\mathrm{Cu}(S=2.6)$.

\begin{tabular}{lcccc}
\hline Sample & Squareness & $\begin{array}{c}\text { Saturation } \\
\text { magnetization, } \\
M_{s}(\mathrm{emu} / \mathrm{g})\end{array}$ & $\begin{array}{c}\text { Coercivity } \\
(\mathrm{G})\end{array}$ & $\begin{array}{c}\text { Retentivity } \\
(\mathrm{emu} / \mathrm{g})\end{array}$ \\
\hline $\begin{array}{l}\mathrm{Ni}-\mathrm{Cu} \\
\text { alloy } \\
(S=2.0)\end{array}$ & 0.326 & 14.695 & 178.84 & 4.791 \\
\hline $\begin{array}{l}\text { Bimetallic } \\
\text { Ni-Cu } \\
(S=2.6)\end{array}$ & 0.305 & 13.565 & 190.82 & 4.134 \\
\hline
\end{tabular}

whether the intergrain exchange exists [13]. Stoner and Wohlfarth have reported that $R=0.5$ suggests randomly oriented noninteracting particles undergoing coherent rotation while $R<0.5$ shows that the particles interact by magnetostatic interaction [14]. The exchange-coupled exists when $R>0.5$. Since the as-synthesized nanoparticles both show values of $R<0.5$ these nanoparticles interact by magnetostatic interaction. $\mathrm{Ni}-\mathrm{Cu}$ alloy $(S=2.0)$ shows greater value of magnetization than bimetallic $\mathrm{Ni}-\mathrm{Cu}(S=$ 2.6). The lower $M_{s}$ value associated to the bimetallic Ni$\mathrm{Cu}$ nanoparticles is attributed to two factors. Firstly, surface distortion is due to oxidation of magnetic bimetallic $\mathrm{Cu}-$ $\mathrm{Ni}$, and this effect is especially prominent due to their large surface-to-volume ratio [15]. Secondly, the magnetocrystalline anisotropy of the nanoparticles depends on the degree of crystallinity of nanoparticles. As observed in XRD, bimetallic $\mathrm{Ni}-\mathrm{Cu}$ is partially crystalline, which suggests that a large portion of the crystals is defect as dislocations can occur within the lattice. This will cause a significant reduction in magnetic moment within the nanoparticles as a result of magnetocrystalline anisotropy distortion. The coercivity, $\mathrm{H}_{c}$, of $\mathrm{Ni}-\mathrm{Cu}$ alloy and bimetallic $\mathrm{Ni}-\mathrm{Cu}$ nanoparticles is 178.84 and $190.82 \mathrm{G}$, respectively. These low values indicate that the magnetic nanoparticles have a tendency to be in a paramagnetic state due to its small diameter. $H_{c}$ is the main technical parameter to characterize the magnetism of magnetic nanoparticles, in which this value is strongly size dependent [16].

\section{Conclusions}

Under mild hydrothermal conditions, $\mathrm{Ni}-\mathrm{Cu}$ nanoalloy with diameter of about $22 \mathrm{~nm}$ was successfully prepared at $95^{\circ} \mathrm{C}$ using Triton X-100 and SDS. Simultaneous reduction and concentration of $\mathrm{Ni}$ and $\mathrm{Cu}$ are vital factors to form $\mathrm{Ni}-\mathrm{Cu}$ nanoalloy. When $S=2.6$, a homogeneous alloy could not be formed due to depletion effect. Ni-Cu nanoalloy shows paramagnetism at $25^{\circ} \mathrm{C}$, which decreased with increased $\mathrm{Ni}$.

\section{Acknowledgments}

G. H. Mohamed Saeed would like to thank Al Neelain University, Sudan for the financial support. He would also like to thank Professor Dr. Abdul Halim Shaari and Ms. Lee Oon Jew of University Putra Malaysia for allowing the use of the VSM facilities. This work is supported by Universiti Kebangsaan Malaysia through Grant no. UKM-OUP-NBT$27-138 / 2008$.

\section{References}

[1] R. E. Rosensweig, Ferrohydrodynamics, Cambridge University Press, Cambridge, Mass, USA, 1985.

[2] J. Ahmed, K. V. Ramanujachary, S. E. Lofland et al., "Bimetallic $\mathrm{Cu}-\mathrm{Ni}$ nanoparticles of varying composition $\left(\mathrm{CuNi}_{3}, \mathrm{CuNi}\right.$, $\mathrm{Cu}_{3} \mathrm{Ni}$ )," Colloids and Surfaces A, vol. 331, no. 3, pp. 206-212, 2008.

[3] H. Akbulut and O. T. Inal, "Plasma-assisted deposition of metal and metal oxide coatings," Journal of Materials Science, vol. 33, no. 5, pp. 1189-1199, 1998.

[4] J. Chatterjee, M. Bettge, Y. Haik, and C. Jen Chen, "Synthesis and characterization of polymer encapsulated $\mathrm{Cu}-\mathrm{Ni}$ magnetic nanoparticles for hyperthermia applications," Journal of Magnetism and Magnetic Materials, vol. 293, no. 1, pp. 303-309, 2005.

[5] D.-H. Kim, S.-H. Lee, K.-N. Kim, K.-M. Kim, I.-B. Shim, and Y.-K. Lee, "Temperature change of various ferrite particles with alternating magnetic field for hyperthermic application," Journal of Magnetism and Magnetic Materials, vol. 293, no. 1, pp. 320-327, 2005.

[6] A. A. Kuznetsov, V. G. Leontiev, V. A. Brukvin et al., "Local radiofrequency-induced hyperthermia using CuNi nanoparticles with therapeutically suitable Curie temperature," Journal of Magnetism and Magnetic Materials, vol. 311, no. 1, pp. 197203, 2007.

[7] W. Mörke, T. Bieruta, J. Jarsetz, C. Görsmann, and U. Schubert, "Characterization of highly dispersed bimetallic Ni$\mathrm{Cu}$ alloy particles by ferromagnetic resonance," Colloids and Surfaces A, vol. 115, pp. 303-309, 1996.

[8] R. G. Samvel'yan, E. S. Abovyan, S. G. Agbalyan, N. N. Manukyan, and M. S. Sakanyan, "X-ray diffraction examination of the process of thermal stabilization of powder thermal bimetals," Soviet Powder Metallurgy and Metal Ceramics, vol. 30, no. 7, pp. 606-609, 1991.

[9] G. Cardenas T. and R. Oliva C., "Synthesis and characterization of bimetallic Ni-Cu colloids," Materials Research Bulletin, vol. 33, no. 11, pp. 1599-1608, 1998.

[10] J. Feng and C.-P. Zhang, "Preparation of Cu-Ni alloy nanocrystallites in water-in-oil microemulsions," Journal of Colloid and Interface Science, vol. 293, no. 2, pp. 414-420, 2006.

[11] Z. Zhang, D. A. Blom, Z. Gai, J. R. Thompson, J. Shen, and S. Dai, "High-yield solvothermal formation of magnetic CoPt alloy nanowires," Journal of the American Chemical Society, vol. 125, no. 25, pp. 7528-7529, 2003.

[12] H. L. Niu, Q. W. Chen, Y. S. Lin, Y. S. Jia, H. F. Zhu, and M. Ning, "Hydrothermal formation of magnetic Ni-Cu alloy nanocrystallites at low temperatures," Nanotechnology, vol. 15, no. 8, pp. 1054-1058, 2004.

[13] Z. L. Wang, Y. Liu, and Z. Zhang, Handbook of Nanophase and Nanostructured Materials Vol. III: Materials Systems and Applications I, Kluwer Academic/Plenum Publishers, New York, NY, USA, 2003.

[14] E. C. Stoner and E. P. Wohlfarth, "A mechanism of magnetic hysteresis in heterogeneous alloys," Philosophical Transactions of the Royal Society A, vol. 240, pp. 599-642, 1948. 
[15] M. Rajendran, R. C. Pullar, A. K. Bhattacharya, D. Das, S. N. Chintalapudi, and C. K. Majumdar, "Magnetic properties of nanocrystalline $\mathrm{CoFe}_{2} \mathrm{O}_{4}$ powders prepared at room temperature: variation with crystallite size," Journal of Magnetism and Magnetic Materials, vol. 232, no. 1-2, pp. 71-83, 2001.

[16] G. Herzer, "Grain size dependence of coercivity and permeability in nanocrystalline ferromagnets," IEEE Transactions on Magnetics, vol. 26, no. 5, pp. 1397-1402, 1990. 

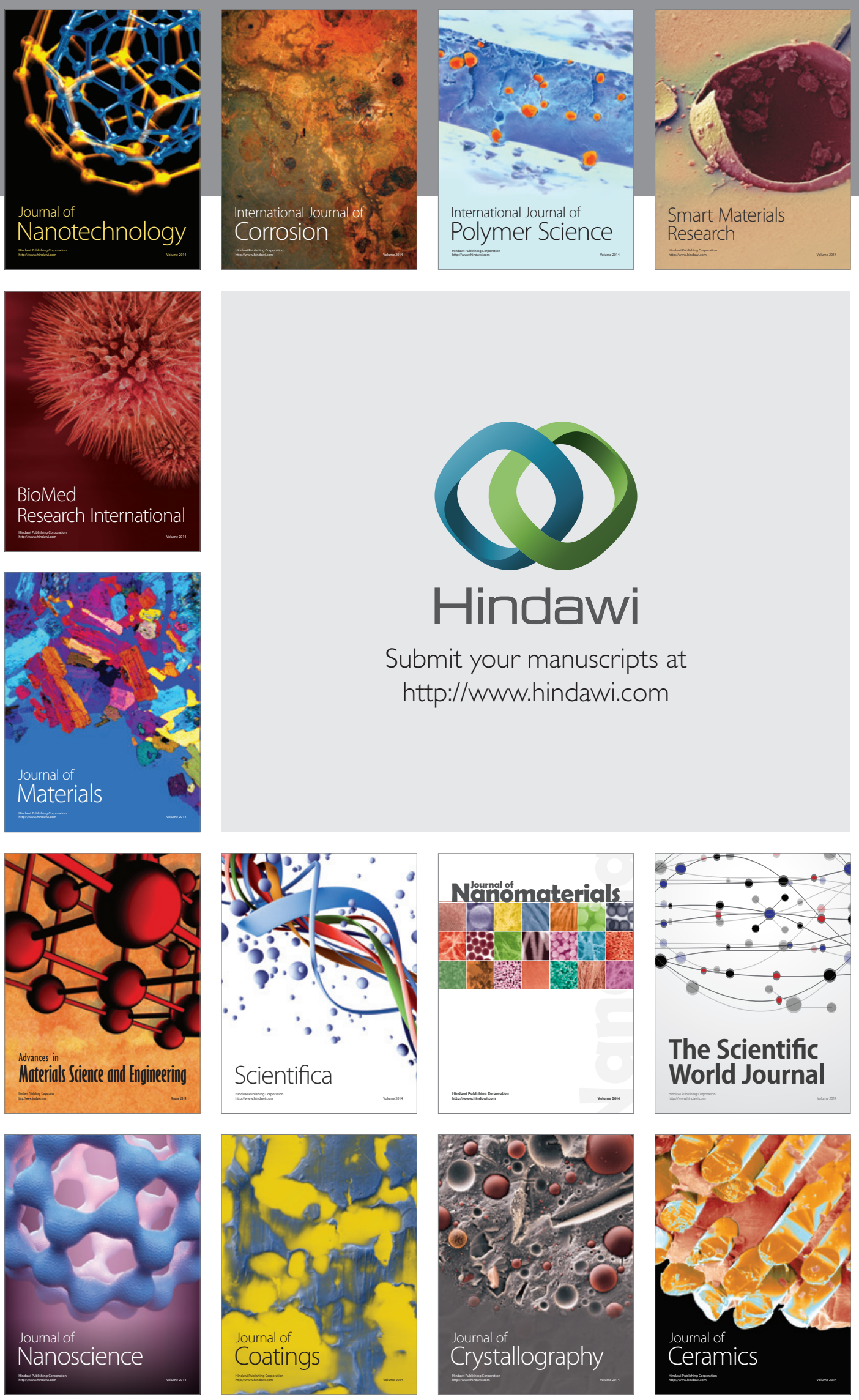

The Scientific World Journal

Submit your manuscripts at

http://www.hindawi.com

\section{World Journal}

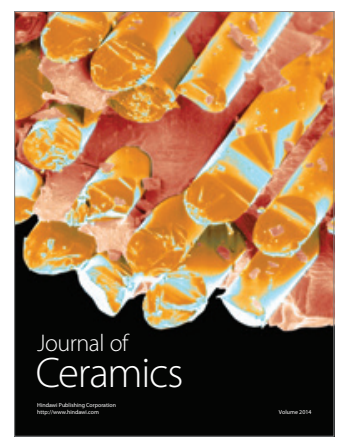

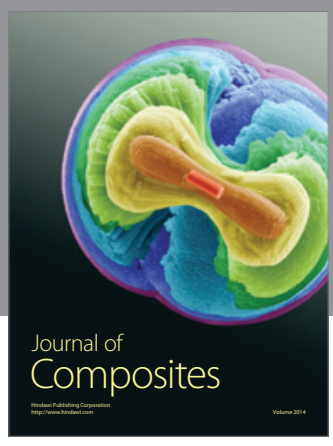
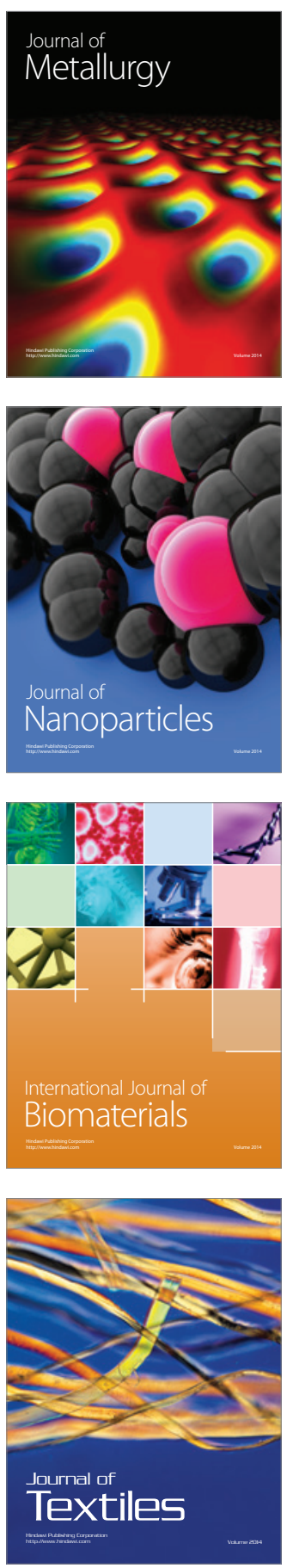